\title{
Global Impact of Heparin on Gene Expression Profiles in Neural Cells Infected by Enterovirus 71
}

\author{
Hamid Reza Pourianfar Enzo Palombo Lara Grollo \\ Environment and Biotechnology Centre, Swinburne University of Technology, Melbourne, Vic., Australia
}

\section{Key Words}

Antiviral activity · DNA microarray · Enterovirus 71 .

Heparin · Molecular mechanism

\begin{abstract}
Objectives: Heparan sulphate mimetics, particularly heparin (Hep), have previously been shown to considerably inhibit infection of enterovirus 71 (EV71) in Vero cells. Therefore, in this study, a genome-wide DNA microarray was performed to gain insight into the mechanism(s) of action of Hep against infection of a human neural cell line, SK-N-SH, with a clinical strain of EV71. Methods: This study focused on a selection of EV71-induced genes whose expression profiling was exclusively affected by the antiviral activity of Hep. The selection procedure was performed through a statistical multi-level comparison with the following controls: negative control cells, compound control (cells treated with Hep only), virus control (cells treated with virus only) and treatment control (EV71-infected cells treated with Hep). Results: Overall, of more than 30,000 genes studied, 14 well-known annotated genes were selected that may be targets for the antiviral activity of Hep against EV71 infection in neural cells. For most of these genes, Hep appeared to modulate the impact of EV71 infection on the expression pattern of the genes. Conclusions: The findings of this research may provide initial assistance in new directions for studies to design molecular drug targets against EV71 infection.

(c) 2013 S. Karger AG, Basel
\end{abstract}

(C) 2013 S. Karger AG, Basel

$0300-5526 / 13 / 0572-0093 \$ 38.00 / 0$

\section{Introduction}

Enterovirus 71 (EV71) infection remains a public health problem at a global level, particularly in the AsiaPacific region. The infection normally manifests as handfoot-mouth disease; however, it is capable of causing potentially fatal neurological complications [1]. Despite several compounds having been reported to exhibit antiviral potencies against EV71 infection, there is still currently no approved vaccine or antiviral substance for prophylaxis or treatment of the infection [2]. Therefore, research studies need to continue to explore potential compounds that exhibit excellent potencies against EV71 infection.

In a previous study, we showed that soluble heparan sulphate (HS)-like substances, including heparin (Hep), pentosan polysulphate and HS, exhibited potent antiviral activities against a cloned strain of EV71 in Vero cells [3]. It was further demonstrated that Hep and pentosan polysulphate significantly prevented attachment of a low-passage clinical strain of EV71 to Vero cells [unpubl. data], results that were further supported with the proved role of cell surface HS in serving as an attachment receptor for EV71 [4]. These observations warranted further investigations in order to explore the molecular mechanisms through which soluble Hep, as an HS-mimetic compound, may inhibit EV71 infection in vitro. Thus, in this study we attempted to gain global

\section{KARGER}

E-Mail karger@karger.com

www.karger.com/int
Hamid Reza Pourianfar

Iranian Academic Centre for Education Culture and Research Mashhad Branch

PO Box 91775-1376, Mashhad (Iran)

E-Mail reza.pourianfar@gmail.com 
insight into the molecular mechanisms of action of Hep in EV71-infected neural cells using a genome-wide microarray analysis.

\section{Materials and Methods}

\section{Cell Lines and Viruses}

A human neuroblastoma cell line, SK-N-SH, from the European Collection of Cell Cultures (catalogue number 86012802) was purchased from CellBank Australia (Westmead, N.S.W., Australia). The cells were maintained in Dulbecco's modified Eagle's medium (Invitrogen, Mulgrave, Vic., Australia) supplemented with 10\% heat-inactivated fetal bovine serum (Invitrogen).

A clinical isolate of EV71 (number 99018233) was supplied from Victorian Infectious Disease Reference Laboratories (North Melbourne, Vic., Australia). The virus was propagated and titrated in $80 \%$ confluent Vero cells as described elsewhere [5].

\section{Reagents}

Heparin sodium salt (Hep) from bovine intestinal mucosa $(\geq 140$ United States Pharmacopeia (USP) units/mg; catalogue number H0777), purchased from Sigma-Aldrich (Castle Hill, N.S.W., Australia), was dissolved in sterile distilled water $(10 \mathrm{mg} / \mathrm{ml})$ as described previously [6].

\section{Cell Seeding and Controls}

Cells were seeded at $1.3 \times 10^{5}$ cells/well in 12-well plates (Becton Dickinson, North Ryde, N.S.W., Australia) followed by incubation at $37^{\circ}$ in $5 \% \mathrm{CO}_{2}$ until $80 \%$ confluent. The following conditions were prepared: (1) negative control, i.e. not infected with the virus or treated with Hep; (2) virus control, i.e. infected with 100 median tissue culture infective dose EV71 but not treated with Hep; (3) compound control, i.e. treated with Hep at $125 \mu \mathrm{g} / \mathrm{ml}$ only, and (4) treatment, i.e. treated with Hep and simultaneously infected with EV71.

Three wells were allocated to each of the above-mentioned conditions; 2 wells were used to assess cytotoxicity and antiviral activity and the third well was allocated to total RNA isolation for that condition. Assessment of cytotoxicity and antiviral activity and RNA isolation were performed at the same time, namely after $48 \mathrm{~h}$, and each was independently replicated 3 times.

\section{Cytotoxicity and Antiviral Activity Assays}

The cytotoxicity and anti-EV71 activity of Hep were assessed using MTT reagent (Invitrogen), followed by incubation for 2-3 h, after which the formazan was dissolved with DMSO. The colour change was recorded using a microplate reader at $540 \mathrm{~nm}$. Cytotoxicity was measured based on the amount of living cells in the compound control relative to the negative control. Antiviral activity was expressed as a percentage of virus inhibition using the following equation [7]: (treatment - virus control)/(negative control - virus control).

\section{RNA Isolation and Quantification}

Total RNA was isolated using the RNeasy Mini Kit according to the manufacturer's instructions (Qiagen Pty. Ltd., Vic., Australia; catalogue number 74104). For each of the 4 conditions described above, 3 independent biological replicates were prepared so that 12 samples went to the hybridization assays.
In order to assess RNA purity and integrity, the RNA samples were initially monitored by agarose (1\%) gel electrophoresis, followed by measurement using the Eukaryote Total RNA Nano assay, version 2.6 (Agilent 2100 Bioanalyzer, Agilent Technologies Inc.). Electropherograms of the total RNA samples with an integrity number over 8.5 were further analysed, and the following criteria were taken into account: at least $20 \%$ of fragmented RNA was located between $28 \mathrm{~S}$ and $18 \mathrm{~S}$ rRNA, the $28 \mathrm{~S}$ and $18 \mathrm{~S}$ peaks account for at least 5 and $10 \%$ of the overall RNA, respectively, and there was no more than $60 \%$ of RNA with fragment sizes smaller than $18 \mathrm{~S}$ rRNA [8]. The total RNA samples that passed these criteria were selected for further labelling.

\section{Arrays}

GeneChip ${ }^{\circledR}$ Human Gene 1.0 ST arrays offering whole-transcript coverage were purchased from Affymetrix (catalogue number 901086). Each microarray chip used in this study contained 33,297 distinct probe sets (transcript cluster IDs), each represented by approximately 26 probes ( 25 -mer) spread across the full length of the gene. The Gene 1.0 ST arrays require sense orientation of labelled cDNA targets.

\section{Target Preparation and Hybridization Process}

The target preparation and hybridization process was performed at the Peter MacCallum Cancer Centre (East Melbourne, Vic., Australia). The Ambion ${ }^{\circledR}$ WT Expression Kit was used to generate sense strand cDNA targets. In brief, $3 \mu \mathrm{l}$ of starting total RNA (300 ng) was mixed with $2 \mu$ of the mix of T7-(N)6 Primers/ Poly-A RNA control solution followed by incubation at $70^{\circ}$ for $5 \mathrm{~min}$ and $4^{\circ}$ for a minimum of $2 \mathrm{~min}$. Following synthesis of the first strand of cDNA, DNA polymerase synthesized the second strand, which served as a template for in vitro transcription, during which anti-sense cRNA was synthesized using T7 RNA polymerase. Then, the second-cycle cDNA (sense strand) was synthesized followed by fragmentation and labelling of sense-stranded cDNA using the Affymetrix GeneChip WT Terminal Labeling Kit. Hybridization was then performed in a hybridization oven at $45^{\circ}$ and $60 \mathrm{rpm}$ for $17 \pm 1 \mathrm{~h}$ followed by washing and scanning of the arrays.

\section{Statistical Analysis}

Since Affymetrix does not add mismatch probes in the newer arrays such as the GeneChip Human Gene 1.0 ST array, background noise was handled by a background correction step during the normalization. Partek Genomics Suite version 6.5 was utilized to import and analyse raw data from the array cells. Using a onestep Tukey's bi-weight method, normalized values of multiple probes for the same gene (at each replication) were summarized into a single value representing the consensus level of expression for that gene. The following statistical contrasts were established: (1) three basic comparisons, i.e. gene expression profiling of the negative control condition was contrasted with those of treatment, virus control and compound control, and (2) two complementary comparisons, i.e. gene expression profiling of the treatment condition was statistically compared with those of virus control and compound control.

For the aforementioned statistical comparisons, a single-factor analysis of variance inside Partek was applied to generate $\mathrm{p}$ values. These $\mathrm{p}$ values were also adjusted by the false discovery rate (FDR) to correct for multiple testing [9]. 
Table 1. Cytotoxicity and antiviral potentiality of Hep in EV71 infection of human neural cells

\begin{tabular}{|c|c|c|c|}
\hline & \multicolumn{3}{|l|}{ Condition } \\
\hline & Cyto & Hep & $\mathrm{VC}$ \\
\hline Initial virus titre ${ }^{\mathrm{a}}, \mathrm{TCID}_{50}$ & - & 100 & 100 \\
\hline Survival $48 \mathrm{~h}$ after inoculation ${ }^{\mathrm{b}}, \%$ & $94.4 \pm 0.23$ & $86.1 \pm 1.8$ & $57 \pm 2.4$ \\
\hline Virus titre inhibition $48 \mathrm{~h}$ after inoculation ${ }^{\mathrm{c}}, \%$ & NA & $95.37 \pm 1.3$ & 0.00 \\
\hline
\end{tabular}

Values represent the average of at least 2 independent experiments from which the standard deviations were calculated. The conditions are detailed in Materials and Methods. Cyto $=$ Compound control; Hep = treatment; $\mathrm{VC}=$ virus control; $\mathrm{TCID}_{50}=$ median tissue culture infective dose; $\mathrm{NA}=$ not applicable because the compound control condition had no virus, while the virus titre inhibition was 0 in the virus control condition due to lack of the virus inhibitor.

a The clinical EV71 isolate was propagated and titrated in $80 \%$ confluent Vero cells as stated in Materials and Methods. The Vero cell-based titrated virus was then used to infect neural cells in both treatment control and virus control conditions.

${ }^{b}$ Survival of the neural cells was calculated based on the amount of living cells in each condition relative to negative control $48 \mathrm{~h}$ after inoculation.

${ }^{\mathrm{c}}$ Virus titre inhibition caused by Hep was determined $48 \mathrm{~h}$ after viral inoculation using the following equation: (treatment - virus control)/(negative control - virus control).

\section{Results}

\section{Cytotoxicity and Antiviral Activity}

Hep at $125 \mu \mathrm{g} / \mathrm{ml}$ significantly prevented in vitro EV71 infection without having a cytotoxic effect on the neural cells (table 1). These results were in agreement with the previous findings, where Hep along with other HS mimetics demonstrated significant anti-EV71 activity with minimal cytotoxicity in Vero cells [3].

\section{Global Profiling of Gene Expression}

Changes in gene expression intensities of the 12 RNA samples of neural cells were independently normalized. Overall, results showed that the histograms of gene expression values of the samples followed the same trend, suggesting the arrays were technically acceptable. The microarray data were then deposited in the National Center for Biotechnology Information's Gene Expression Omnibus, accessible through the accession number GSE34234 (online at http://www.ncbi.nlm.nih.gov/geo/ query/acc.cgi?acc=GSE34234).

\section{Impact of Antiviral Activity of Hep on Gene} Expression of EV71-Infected Cells

A multi-level selection procedure was employed in order to attribute changes at the gene transcription level to the antiviral activity of Hep. In order to simplify this procedure, we began with statistical comparisons between two conditions, namely treatment and virus control. In this way, it was possible to statistically extract the effect of the virus on neural cells from the antiviral effect of Hep on EV71-infected neural cells. Choosing a cut-off $\mathrm{p}$ value of less than 0.05 with fold changes equal to or more than 1.5 (up- or down-regulation) resulted in selection of 144 genes that were differentially expressed between the conditions. These 144 selected genes were further monitored in the condition of virus control to assess how significantly their expression was affected by EV71 infection. As a result, 14 genes (of 144 genes) were found to be significantly induced by EV71 infection at a p value less than 0.05 and an FDR-adjusted p value lower than 0.48 with a fold change of at least 1.5 (up- or down-regulation; table 2).

In the next step, we examined whether gene expression changes attributed to the antiviral activity of Hep might have been due to the general effect of Hep as a substance. Hence, the expression patterns were assessed in the condition of compound control, showing that Hep alone had no significant inductive effect on the 14 selected genes ( $p>0.05$ and/or a fold change less than 1.5), except for the basic leucine zipper transcription factor, ATF-like gene, which was thus excluded from further bioinformatics analysis (table 2).

A further selection approach revealed 5 other genes that were not significantly induced by EV71 infection (virus control) or Hep alone (compound control) but demonstrated considerable induction in the treatment condition as compared to negative control (table 3). Consequently, there were significant differences in the expression pattern of these genes between the treatment condition and virus control or compound control ( $\mathrm{p}<$ 0.05 , with a fold change more than 1.5). Thus, these genes were also chosen for further bioinformatics characterization. 

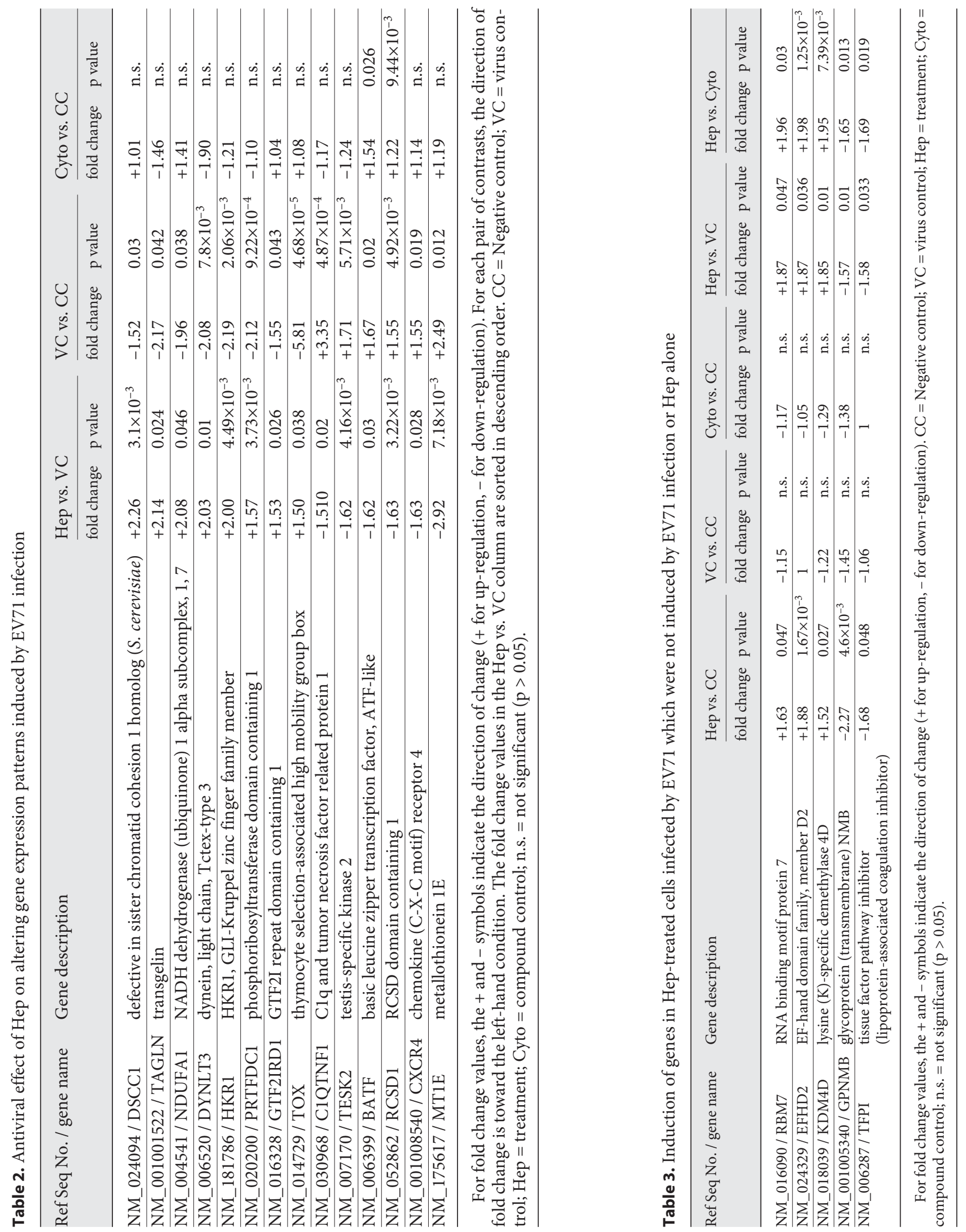
Table 4. Antiviral effect of Hep on altering expression patterns of un-annotated genes induced by EV71 infection

\begin{tabular}{|c|c|c|c|c|c|c|}
\hline \multirow[t]{2}{*}{ Cluster transcript ID } & \multicolumn{2}{|l|}{ Hep vs. VC } & \multicolumn{2}{|l|}{ VC vs. CC } & \multicolumn{2}{|l|}{ Cyto vs. CC } \\
\hline & fold change & $\mathrm{p}$ value & fold change & $\mathrm{p}$ value & fold change & $\mathrm{p}$ value \\
\hline 7892904 & +2.34 & $5.27 \times 10^{-3}$ & -2.12 & $7.93 \times 10^{-3}$ & +1.26 & n.s. \\
\hline 7893771 & +1.86 & 0.039 & -2.15 & $8.49 \times 10^{-3}$ & -1.03 & n.s. \\
\hline 7895000 & +1.81 & 0.033 & -1.84 & 0.031 & -1.09 & n.s. \\
\hline 7892628 & +1.72 & 0.037 & -2.29 & $2.45 \times 10^{-3}$ & -1.29 & n.s. \\
\hline 7896113 & +1.67 & $2.87 \times 10^{-3}$ & -1.75 & 0.019 & -1.35 & n.s. \\
\hline 7894973 & +1.64 & $2.98 \times 10^{-3}$ & -1.64 & 0.034 & +1.48 & n.s. \\
\hline 7896426 & +1.57 & 0.039 & -2.02 & 0.018 & -1.30 & n.s. \\
\hline 7895836 & +1.56 & 0.025 & -1.50 & 0.041 & +1.24 & n.s. \\
\hline 7893253 & -1.51 & 0.031 & +1.55 & 0.019 & +1.40 & $5.88 \times 10^{-3}$ \\
\hline 7894742 & -1.61 & 0.019 & +1.65 & $7.56 \times 10^{-3}$ & +1.37 & n.s. \\
\hline 7896256 & -1.62 & 0.037 & +1.65 & 0.025 & +1.26 & n.s. \\
\hline 7894614 & -1.76 & $8.48 \times 10^{-3}$ & +1.78 & 0.015 & +1.16 & n.s. \\
\hline 7893180 & -2.83 & $2.02 \times 10^{-3}$ & +1.82 & 0.011 & -1.05 & n.s. \\
\hline
\end{tabular}

For fold change values, the + and - symbols indicate the direction of change (+ for up-regulation, - for downregulation). $\mathrm{CC}=$ Negative control; $\mathrm{VC}=$ virus control; Hep $=$ treatment; Cyto $=$ compound control; n.s. $=$ not significant $(\mathrm{p}>0.05)$.

In addition to the well-annotated genes listed in tables 2 and 3, the same selection procedure was also employed to select genes for which no annotation was found using the NetAffx annotation pipeline (table 4).

The results of this study also revealed that there were more than 1,000 other genes induced by EV71 infection in the condition of virus control with expression changes that ranged from +46.5 - to -10.7 -fold at significance levels lower than 0.01 and an FDR-adjusted p value lower than 0.33 . However, since these changes were not related to the antiviral activity of Hep, they were not further analysed in the current study but will be investigated in future studies.

In addition to the quantifiable comparisons stated above, figure 1 graphically visualizes comparisons across the 4 conditions, i.e. treatment, virus control, compound control and negative control. These illustrative comparisons were drawn based on the same positions (features) on the chips belonging to one of the replicons.

\section{Discussion}

There are numerous studies using DNA microarrays to explore the mechanisms of action of commercial antiviral drugs such as interferons in human cell lines [10, 11]. However, there has been minimal investigation into the global impact of a purported antiviral compound on gene expression of cells that have been infected with the desired virus. Among the few examples, microarray analysis was used to reveal changes to the gene expression pattern of Alexander cells secreting hepatitis B virus surface antigen that were treated with an ethanolic extract of Phyllanthus nanus [12]. In addition, microarray analysis of a monoclonal antibody showed that it prevented hepatitis $\mathrm{C}$ virus infection through the betaine/GABA transporter-1 gene [13]. Such studies with EV71 are even more limited, as the only reported large-scale microarray analysis of EV71 detailed expression patterns of a human glioblastoma cell line following virus infection [14]. To this effect, there has been no study reported in the literature to assess the mechanisms of action of antiviral compounds against EV71 infection using DNA microarray analysis.

Overall, in this study 14 well-annotated genes were found whose EV71-induced expression profiles were modified by Hep, leading to a substantial difference ( $>1.5$-fold change, $\mathrm{p}<0.05)$ between the conditions of treatment and virus control. In fact, Hep lessened the intensities of EV71-mediated down-regulation or upregulation of these genes. In the following, we discuss the selected genes for which we were able to make speculations, while we could not draw conclusions for the rest. 


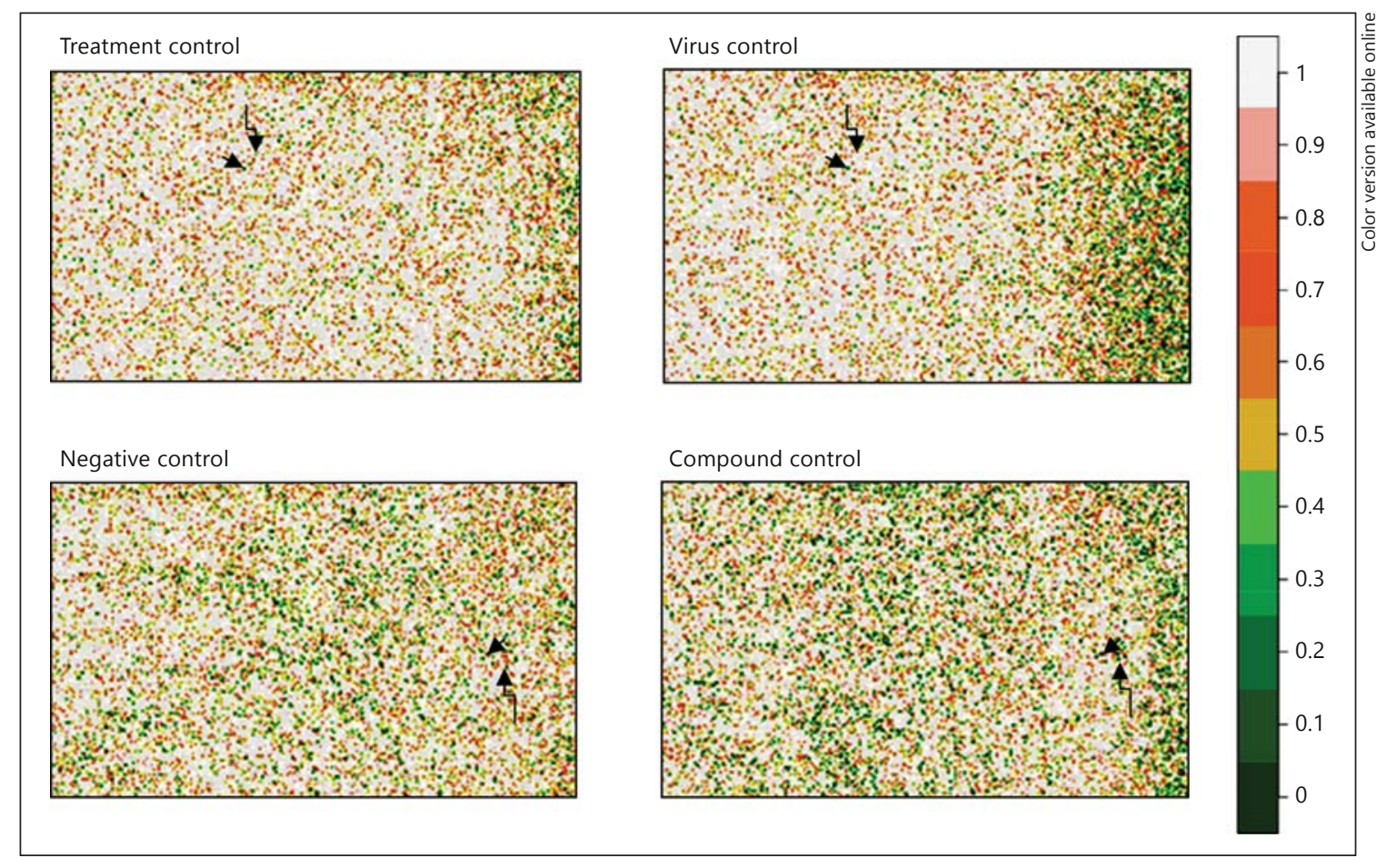

Fig. 1. Images of microarray analysis of 4 conditions. The images illustrate small parts of each chip and represent how the genes are differentially expressed across different conditions. The colour intensity (right) shows different gene expression values, such that dark-green and white represent up-regulated and down-regulated genes, respectively (colours refer to the online version only). To make comparisons simpler, the same features are compared between treatment and virus control, and other (same) features are compared between negative control and compound control. The same expression intensity is indicated with straight arrows, while differentially expressed features are indicated with elbow arrows.

\section{Group 1: EV71-Suppressed Genes Whose Expression Was Altered by Hep \\ DSCC1}

In eukaryotes, DSCC1, along with chromosome transmission fidelity factor 18 homolog (S. cerevisiae) (CHTF18) and chromosome transmission fidelity factor 8 homolog (S. cerevisiae) (CHTF8), plays an essential role in cell proliferation [15]. As such, mutations in any one of these genes are believed to result in growth arrest and ultimately cell death [16-18]. In this study, EV71mediated down-regulation of DSCC1 may thus have resulted in increased cell death, while this effect was counteracted by the addition of Hep.

\section{TAGLN}

Disruption of the cytoplasmic organization of microfilaments during virus infection has been linked to a down-regulation of TAGLN along with other genes involved in the assembly and disassembly of actin fibers, leading to observation of a viral cytopathic effect [19].
Therefore, Hep could possibly negate an EV71-mediated cytopathic effect by modifying the negative impact of EV71 on expression of TAGLN.

\section{NDUFA1}

Recently, evidence was provided for a role of NDUFA1 and other genes of respiratory chain complexes in regulating coxsackievirus B3-mediated myocarditis in vivo. Decreased expression of respiratory chain complexes led to suppression of both oxidative stress and mitochondrial apoptosis, increasing viral replication in the heart [20]. In the context of this in vitro study, it could be suggested that Hep might have assisted a mitochondrial intracellular antiviral state by neutralization of EV71-induced down-regulation of NDUFA1.

\section{GTF2IRD1}

GTF2IRD1 has been shown to play an important role in cellular processes and cell cycle regulation [21]. As such, it could be proposed that EV71-mediated down- 
regulation of GTF2IRD1 may have affected cellular processes such as signaling and the cell cycle, which would benefit progression of the infection; this affect appeared to be neutralized by Hep.

\section{Group 2: EV71-Up-Regulated Genes Whose \\ Expression Was Altered by Hep \\ TESK2}

It has been demonstrated that expression of TESK2 in HeLa cells was involved in actin cytoskeletal re-organization by phosphorylating and regulating cofilin/actin-depolymerizing factor. In addition, a TESK2 mutant with excess phosphorylation activity caused condensation and fragmentation of nuclei followed by apoptosis in HeLa cells [22]. Thus, these findings may have an implication for the current investigation in that the EV71-mediated increase of TESK 2 would ultimately lead to the death of neural cells. Again, Hep might have modulated these events through a decrease in the up-regulation of the protein.

\section{CXCR4}

Hep suppressed EV71-induced expression of CXCR4 receptor, which is 1 of the 7 known CXC chemokine receptors in many mammalian cells [23]. In addition, this receptor together with CCR5 is utilized by HIV-1 to gain entry into target cells [24]. Up-regulation of CCR5 following EV71 infection in glioblastoma cells was reported [14]. However, use of CXCR4 receptor by EV71 has not been reported to date. It remains to investigate whether EV71 penetration would be hindered by the effect of Hep on EV71-induced up-regulation of CXCR4.

Apart from the genes discussed above, we found that heparan sulphate 2-O-sulfotransferase 1 (HS2ST1) was significantly up-regulated by EV71 (+1.81-fold). This protein is responsible for the biosynthesis of cell surface HS. Addition of Hep to EV71-infected neural cells did not significantly change the expression of HS2ST1 compared to virus control. This initially meant that Hep did not have any effect on the induction of HS2ST1. However, these findings provide evidence that clinical EV71 needs cell surface HS to infect or bind neural cells, in agreement with previous results from cell culture [3,4]. Considering this, it would then be reasonable to suppose that an increase in the level of HS2ST1 by EV71 may be masked by competitive electrostatic interaction between soluble Hep and the EV71 capsid that ultimately leads to prevention of viral infection or attachment.

In this study, we also found genes that were not significantly induced by EV71 infection or Hep alone but were seen to be changed in the treatment condition. Thus, we proposed that Hep in the presence of EV71 might have altered the expression pattern of these genes in order to prevent viral infection. We limited our analysis to GPNMB, for which there was adequate information in the literature. Hep suppressed EV71-induced expression of GPNMB significantly in comparison with both virus control and compound control. GPNMB encodes the glycoprotein (transmembrane) NMB, which serves as a feedback regulator of pro-inflammatory responses [25]. A lymphatic tissue microarray analysis showed an increase in several immunosuppressive genes including GPNMB during HIV-1 infection [26]. Similar results were gained with pigs infected by porcine circovirus type 2 [27]. These findings might suggest a possible molecular target for antiviral activity of Hep in vivo, where lymph nodes would be infected with EV71.

One of the possibilities suggested by the previous findings $[3,4]$ was that electrostatic interaction between the negatively charged Hep and the positively charged viral capsid of EV71 could interfere with EV71 attachment or entry. Hence, it should not be ruled out that changes to EV71-induced genes seen in this microarray analysis might be partially attributable to a significant inhibitory effect of Hep on EV71 entry into the cells. However, the EV71-induced genes whose expression was seen to be changed by Hep might still suggest molecular targets for further studies to design antiviral drugs against EV71. Furthermore, EV71 attachment was not completely blocked by Hep in Vero cells [3] or human neural cells (data not shown). As a result, EV71-mediated induction of gene expression in the host cells could have been counteracted by the presence of Hep. To the best of our knowledge, this is the first microarray analysis of an anti-EV71 compound through a multi-level comparison such that the microarray data were directly linked to the findings of antiviral activity and cytotoxicity tests.

\section{Acknowledgements}

This work was funded by a Grollo Ruzzene Foundation grant awarded to Dr. L. Grollo. The authors wish to thank Dr. Julian Druce of the Victorian Infectious Disease Reference Laboratories (Parkville, Vic., Australia) for providing the clinical strain of EV71. We also thank Drs. Richard Tothil, Tim Holloway, Li Jason, Aga Borcz and other colleagues of the Molecular Genomics Core Facility at the Peter MacCallum Cancer Centre (East Melbourne, Vic., Australia) for processing the microarray hybridization assays and assistance in analysing microarray data. 


\section{References}

1 McMinn PC: An overview of the evolution of enterovirus 71 and its clinical and public health significance. FEMS Microbiol Rev 2002;26:91-107.

-2 Shang L, Xu M, Yin Z: Antiviral drug discovery for the treatment of enterovirus 71 infections. Antiviral Res 2013;97:183-194.

$>3$ Pourianfar HR, Poh CL, Fecondo J, Grollo L: In vitro evaluation of the antiviral activity of heparan sulfate mimetic compounds against Enterovirus 71. Virus Res 2012;169:22-29.

-4 Tan CW, Poh CL, Sam IC, Chan YF: Enterovirus 71 uses cell surface heparan sulfate glycosaminoglycan as an attachment receptor. J Virol 2013;87:611-620.

5 Pourianfar HR, Javadi A, Grollo L: A colorimetric-based accurate method for the determination of Enterovirus 71 titer. Indian J Virol 2012;23:303-310.

-6 Zautner AE, Korner U, Henke A, Badorff C, Schmidtke M: Heparan sulfates and coxsackievirus-adenovirus receptor: each one mediates coxsackievirus B3 PD infection. J Virol 2003;77:10071-10077.

-7 Schmidtke M, Schnittler U, Jahn B, Dahse HM, Stelzner A: A rapid assay for evaluation of antiviral activity against coxsackie virus B3, influenza virus $\mathrm{A}$, and herpes simplex virus type 1. J Virol Methods 2001;95:133-143.

>8 Schoor O, Weinschenk T, Hennenlotter J, Corvin S, Stenzl A, Rammensee HG, Stevanović S: Moderate degradation does not preclude microarray analysis of small amounts of RNA. BioTechniques 2003;35:1192-1201.

9 Benjamini Y, Hochberg Y: Controlling the false discovery rate: a practical and powerful approach to multiple testing. J R Stat Soc B 1995;57:289-300.

10 Der SD, Zhou A, Williams BR, Silverman RH: Identification of genes differentially regulated by interferon alpha, beta, or gamma using oligonucleotide arrays. Proc Natl Acad Sci USA 1998;95:15623-15628.

>11 Domingo-Gil E, González JM, Esteban M: Identification of cellular genes induced in human cells after activation of the OAS/RNaseL pathway by vaccinia virus recombinants expressing these antiviral enzymes. J Interferon Cytokine Res 2010;30:171-188.
12 Lam WY, Leung KT, Law PTW, Lee SMY, Chan HLY, Fung KP, Ooi VEC, Waye MMY: Antiviral effect of Phyllanthus nanus ethanolic extract against hepatitis B virus (HBV) by expression microarray analysis. J Cell Biochem 2006;97:795-812.

-13 Satoh M, Saito M, Takano T, Kasama Y, Nishimura T, Nishito Y, Hirata Y, Arai M, Sudoh M, Kai C, Kohara M, Tsukiyama-Kohara K: Monoclonal antibody 2-152a suppresses hepatitis $\mathrm{C}$ virus infection through betaine/ GABA transporter-1. J Infect Dis 2011;204: 1172-1180.

14 Shih SR, Stollar V, Lin JY, Chang SC, Chen GW, Li ML: Identification of genes involved in the host response to enterovirus 71 infection. J Neurovirol 2004;10:293-304.

15 Bermudez VP, Maniwa Y, Tappin I, Ozato K, Yokomori K, Hurwitz J: The alternative Ctf18-Dcc1-Ctf8-replication factor C complex required for sister chromatid cohesion loads proliferating cell nuclear antigen onto DNA. Proc Natl Acad Sci USA 2003;100: 10237-10242.

16 Atienza JM, Roth RB, Rosette C, Smylie KJ, Kammerer S, Rehbock J, Ekblom J, Denissenko MF: Suppression of RAD21 gene expression decreases cell growth and enhances cytotoxicity of etoposide and bleomycin in human breast cancer cells. Mol Cancer Ther 2005;4: 361-368.

17 Ghiselli G: SMC3 knockdown triggers genomic instability and p53-dependent apoptosis in human and zebrafish cells. Mol Cancer 2006;5:52-64.

18 Gluenz E, Sharma R, Carrington M, Gull K: Functional characterization of cohesin subunit SCC1 in Trypanosoma brucei and dissection of mutant phenotypes in two life cycle stages. Mol Microbiol 2008;69:666-680.

19 Hertel L, Mocarski ES: Global analysis of host cell gene expression late during cytomegalovirus infection reveals extensive dysregulation of cell cycle gene expression and induction of pseudomitosis independent of US28 function. J Virol 2004;78:11988-12011.
20 Ebermann L, Wika S, Klumpe I, Hammer E, Klingel K, Lassner D, Völker U, Erben U, Zeichhardt H, Schultheiss HP, Dörner A: The mitochondrial respiratory chain has a critical role in the antiviral process in Coxsackievirus B3-induced myocarditis. Lab Invest 2012;92: 125-134.

21 Chimge NO, Makeyev AV, Ruddle FH, Bayarsaihan D: Identification of the TFII-I family target genes in the vertebrate genome. Proc Natl Acad Sci USA 2008;105:90069010.

22 Toshima J, Toshima JY, Takeuchi K, Mori R, Mizuno K: Cofilin phosphorylation and actin reorganization activities of testicular protein kinase 2 and its predominant expression in testicular Sertoli cells. J Biol Chem 2001;276: 31449-31458.

23 Bajetto A, Bonavia R, Barbero S, Piccioli P, Costa A, Florio T, Schettini G: Glial and neuronal cells express functional chemokine receptor CXCR4 and its natural ligand stromal cell-derived factor 1. J Neurochem 1999;73: 2348-2357.

24 Juffermans NP, Paxton WA, Dekkers PEP, Verbon A, De Jonge E, Speelman P, Van Deventer SJH, Van Der Poll T: Up-regulation of HIV coreceptors CXCR4 and CCR5 on CD4+ $\mathrm{T}$ cells during human endotoxemia and after stimulation with (myco)bacterial antigens: the role of cytokines. Blood 2000;96:26492654.

25 Ripoll VM, Irvine KM, Ravasi T, Sweet MJ, Hume DA: Gpnmb is induced in macrophages by IFN- $\gamma$ and lipopolysaccharide and acts as a feedback regulator of proinflammatory responses. J Immunol 2007;178:65576566.

26 Li Q, Smith AJ, Schacker TW, Carlis JV, Duan L, Reilly CS, Haase AT: Microarray analysis of lymphatic tissue reveals stage-specific, gene expression signatures in HIV-1 infection. J Immunol 2009;183:1975-1982.

27 Lee G, Han D, Song JY, Lee YS, Kang KS, Yoon S: Genomic expression profiling in lymph nodes with lymphoid depletion from porcine circovirus 2-infected pigs. J Gen Virol 2010;91:2585-2591. 